\title{
Association between thyroid hormones and the components of metabolic syndrome
}

\author{
Jieun Jang ${ }^{1,2+}$, Youngsook Kim ${ }^{3 \dagger}$, Jaeyong Shin ${ }^{2,4}$, Sang Ah Lee ${ }^{1,2}$, Young Choi ${ }^{1,2}$ and Eun-Cheol Park ${ }^{2,4^{*}}$ (D)
}

\begin{abstract}
Background: Thyroid hormones are known to have direct and indirect effects on metabolism. Individuals with metabolic syndrome, a disease that is growing in incidence at a rapid rate, are at higher risk for cardiovascular disease, diabetes, and cancer. The aim of this study was to identify whether significant correlations exist between thyroid hormone levels and components of the metabolic syndrome in the general population of Korea.

Methods: The data were collected from the sixth Korea National Health and Nutrition Examination Surveys from 2013 to 2015. A total of 1423 participants were tested for thyroid function. The analysis of variance and multiple linear regression were performed to analyze the relationship between thyroid hormone level and components of the metabolic syndrome.

Results: A positive association between free thyroxine and fasting glucose level was observed in patients with high free thyroxine levels $(\geq 1.70 \mathrm{ng} / \mathrm{dL}, \beta=15.992, p=<0.0001)$, when compared with patients with normal-middle free thyroxine levels. Moreover, a negative association was observed between free thyroxine and triglyceride levels in patients with normal-high free thyroxine levels $(\beta=-21.145, p=0.0054)$ and those with high free thyroxine levels ( $\beta=-49.713, p=0.0404)$.

Conclusion: Free thyroxine shows a partially positive association with fasting glucose and a partially negative association with triglycerides in the Korean population. In patients with abnormal thyroid function, follow up tests for glucose levels and lipid profiling during treatment for thyroid dysfunction would be beneficial in terms of overlooking metabolic syndrome and to prevent related diseases.
\end{abstract}

Keywords: Thyroid hormone, Metabolic syndrome, Free thyroxine, FT4

\section{Background}

Metabolic syndrome, a well-known cluster of cardiovascular risk factors, is a major public health concern worldwide [1, 2]. Metabolic syndrome increases the risk for cardiovascular disease, diabetes, and even certain types of cancer [3]. According to the National Cholesterol Education Program's Adult Treatment Panel III definition, metabolic syndrome is the presence of abnormal values for at least three of the following criteria: waist circumference, serum triglycerides, high-density

\footnotetext{
* Correspondence: ECPARK@yuhs.ac

†ieun Jang and Youngsook Kim contributed equally to this work.

Jieun Jang and Youngsook Kim are co-first authors.

${ }_{2}^{2}$ Institute of Health Services Research, Yonsei University, Seoul, Republic of Korea

${ }^{4}$ Department of Preventive Medicine \& Institute of Health Services Research Yonsei University College of Medicine, 50 Yonsei-ro, Seodaemun-gu, Seoul 120-752, Republic of Korea

Full list of author information is available at the end of the article
}

lipoprotein (HDL) cholesterol, blood pressure, and fasting glucose [3]. The prevalence of metabolic syndrome is increasing rapidly [4]. According to data from the National Health and Nutrition Examination Survey (NHANES) 2011-2012, about 34.7\% of US adults were estimated to have metabolic syndrome [4]. Similar increasing trends have been observed in Europe and other countries $[2,5]$. According to the National Cholesterol Education Program's Adult Treatment Panel III criteria, and per the World Health Organization Asia-Pacific guidelines, the prevalence of metabolic syndrome was approximately $28.2 \%$ in the general population of Korea in 2012 [6]. The mortality rate due to cardiovascular disease has increased from 35.6 to 52.4 out of 100,000 persons over 2003-2014. Thus, more focus and effort is needed to reduce the prevalence of metabolic syndrome,

(C) The Author(s). 2018 Open Access This article is distributed under the terms of the Creative Commons Attribution 4.0 International License (http://creativecommons.org/licenses/by/4.0/), which permits unrestricted use, distribution, and reproduction in any medium, provided you give appropriate credit to the original author(s) and the source, provide a link to the Creative Commons license, and indicate if changes were made. The Creative Commons Public Domain Dedication waiver (http://creativecommons.org/publicdomain/zero/1.0/) applies to the data made available in this article, unless otherwise stated. 
while considering the rapidly increasing frequency of mortality due to related diseases [7].

Thyroid hormones have an important role in metabolism [8]. Abnormal levels of thyroid hormones alter metabolism, and some of these changes share common pathophysiologic processes with metabolic syndrome. Therefore, thyroid dysfunction can affect metabolic syndrome. Lambadiari et al. reported that thyroid hormones are significant determinants of glucose homeostasis [9], and affect fasting glucose levels by antagonizing insulin action [9]. For instance, hyperthyroidism leads to impaired insulin secretion, which suppresses hepatic glucose production and promotes glucose uptake in the muscle [9]. Similarly, Dimitriadis et al. showed that the increased glucose levels in hyperthyroidism could be explained by an increase in endogenous glucose production via of gluconeogenesis [10]. Studies have also demonstrated an association between thyroid hormones and glucose levels. Klein et al. reviewed several studies regarding the mechanism of action of thyroid hormones on the cardiovascular system [11]. They concluded that thyroid hormones have direct and indirect impacts on the cardiovascular system. Patients with thyroid disease, especially hyperthyroidism, often demonstrated signs and symptoms of cardiovascular changes [11]. Several other studies have shown that overt hypothyroidism induces an increase in blood pressure and plasma cholesterol levels [12]. However, most of the studies that assessed the relationships between abnormal thyroid hormone levels and metabolic syndrome have been conducted in Caucasian populations [2, 13]. Therefore, the aim of this study was to assess the relationships between thyroid hormone levels and metabolic syndrome components in a nationally representative sample of South Korean adults.

\section{Methods}

\section{Study population}

This study was conducted using data from the sixth Korea National Health and Nutrition Examination Surveys (KNHANES VI, 2013-2015), a nationwide cross-sectional survey conducted by the Korean Centers for Disease Control and Prevention (Seoul, Korea) to assess the health and nutritional status of the South Korean population. The institutional review board of the Korea Centers for Disease Control and Prevention (KCDC) approved the study (IRB: 2013-07CON-03-4C, 2013-12EXP-03-5C, 201501-02-6C). A nationally representative sample was obtained using a stratified multistage cluster sampling design. The survey consists of a health interview, nutrition survey, and health examination. Tests for thyroid disease and metabolic syndrome components (waist circumference, triglycerides, HDL cholesterol, blood pressure, and fasting glucose) were part of the health examination. The initial sample included 22,948 participants. The thyroid functions tests were carried out by subsampling 2400 subjects aged $\geq 10$ years with respect to thyroid-stimulating hormone (TSH), free thyroxine (FT4), thyroid peroxidase antibody (TPOab), and urinary iodine in the sixth Korea National Health and Nutrition Examination Surveys (KNHANES VI, 2013-2015). Participants with missing thyroid function data were excluded $(n=$ 20,591). Patients who received treatment that could interfere with the test results for thyroid hormone levels and various components of metabolic syndrome, such as radioactive iodine treatment, antithyroid drugs, thyroid hormones, other medication for thyroid disease, dyslipidemia medications, blood pressure regulators, insulin, or glucose regulators were also excluded $(n=429)$. The data for a total of 1423 participants without any missing variables were finally included in the analysis.

\section{Dependent variables and variables of interest}

The dependent variables were the components of metabolic syndrome. Waist circumference was measured at the narrowest spot between the lowest rib and the highest lateral border of the right iliac crest. Systolic blood pressure was measured after the participants relaxed for 5 min while sitting. Triplicate measurements of systolic blood pressure were obtained. The mean of the second and third measured value was used in the analysis. Triglycerides, HDL cholesterol, and fasting glucose levels were measured using the same Hitachi Automatic Analyzer 7600-210 (Hitachi, Tokyo, Japan).

The variable of interest in this study was thyroid hormone levels. Serum FT4, serum TSH, and TPOab levels were measured using an electro-chemiluminescence immunoassay (Cobas: Roche Diagnostics, Penzberg, Germany). Samples were sent to the central certified laboratory and analyzed. The laboratory reference ranges for FT4, TSH, and TPOab were 0.80-1.70 ng/dL, 0.50-5.0 $\mathrm{uIU} / \mathrm{mL}$, and $<34 \mathrm{IU} / \mathrm{mL}$ [14-16]. FT4 levels were divided into five categories: (1) low; under normal $(<0.80 \mathrm{ng} / \mathrm{dL})$; (2) normal-low (<1.17 ng/dL); (3) normal-middle (< $1.31 \mathrm{ng} / \mathrm{dL})$; (4) normal-high (<1.70 ng/dL); and (5) high; upper normal ( $\geq 1.70 \mathrm{ng} / \mathrm{dL}$ ). TSH levels were divided as follows: low; under normal $(<0.50 \mathrm{uIU} / \mathrm{mL})$; normal-low $(<1.80 \mathrm{uIU} / \mathrm{mL}), \quad$ normal-middle $(<2.87 \mathrm{uIU} / \mathrm{mL})$, normal-high $(<5.00 \mathrm{uIU} / \mathrm{mL})$ and high; upper normal $(\geq 5.00 \mathrm{uIU} / \mathrm{mL})$. TPOab levels were categorized as normal $(<34 \mathrm{IU} / \mathrm{mL})$, high with low FT4 $(34 \mathrm{IU} / \mathrm{mL} \leq$ TPOab; FT4 $<1.24 \mathrm{ng} / \mathrm{dL})$, high with high FT4 $(34 \mathrm{IU} / \mathrm{mL} \leq$ TPOab; $1.24 \mathrm{ng} / \mathrm{dL} \leq \mathrm{FT} 4)$. In addition, we categorized TPOab levels into normal $(<34 \mathrm{IU} / \mathrm{mL})$, high with low 
TSH $(34 \mathrm{IU} / \mathrm{mL} \leq \mathrm{TPOab} ; \mathrm{TSH}<2.25 \mathrm{uIU} / \mathrm{mL})$, high with high TSH $(34 \mathrm{IU} / \mathrm{mL} \leq$ TPOab; $2.25 \mathrm{uIU} / \mathrm{mL} \leq \mathrm{FT} 4)$. Considering that the TPOab titer is related to both hypothyroidism and hyperthyroidism, patients with high levels were divided into two groups using two median FT4 levels and two TSH levels.

\section{Covariates}

We adjusted for the covariates of sociodemographic factors, socioeconomic factors, health-behavior factors, and health-condition factors. Sociodemographic factors included age (19-44 years, 45-64 years, > 64 years) and gender (male and female). Socioeconomic factors included educational level (elementary school or less, middle school, high school, and college or over), marital status (married and cohabit, married but no cohabit or bereaved or divorced, and unmarried), household income level (divided into quartiles), region (urban or rural), and occupation (white collar, pink collar, blue collar, and unemployed or else). Urban areas included capitals and metropolitan cities and rural areas comprised the remaining areas. Alcohol consumption (ever or never), smoking (ever or never), and walking activity (active or inactive) were the health-behavior factors. Health-condition factors involved stress level (high, middle, low).

\section{Statistical analysis}

Statistical analysis was performed using the SAS software, version 9.4 (SAS Institute, Cary, NC, USA). All analysis incorporated weights. The mean values and standard deviations of the components of metabolic syndrome were compared using the analysis of variance. Multiple linear regression was performed to analyze the relationship between thyroid hormone level and the components of metabolic syndrome. Subgroup analysis was performed according to age and gender. A $p$-value $<0.05$ was considered to indicate a statistically significant result.

\section{Results}

\section{Demographic characteristics}

Table 1 presents the general characteristics of the study populations. There were 683 males $(48.0 \%)$ and $740 \mathrm{fe}-$ males $(52.0 \%)$ included in this study. Of the 1423 participants, 870 (61.1\%) were aged between 19 and 44 years, 471 participants $(33.1 \%)$ were aged between 45 and 64 years, and 82 participants $(5.8 \%)$ were aged over 64 years. The mean waist circumference was $31.9 \pm$ $4.0 \mathrm{in}$. and the mean triglycerides level was $133.0 \pm$ $120.5 \mathrm{mg} / \mathrm{dL}$. The mean HDL cholesterol level was 52.0 $\pm 12.8 \mathrm{mg} / \mathrm{dL}$, mean blood pressure $114.2 \pm 15.1 \mathrm{mmHg}$, and the mean fasting glucose level $95.0 \pm 16.6 \mathrm{mg} / \mathrm{dL}$. When analyzed according to FT4 levels, waist circumference, triglycerides, and fasting glucose results were statistically significantly different $(p<0.05)$. Patients with high FT4 levels demonstrated considerably higher fasting glucose levels (High: $109.4 \pm 45.2 \mathrm{mg} / \mathrm{dL}$; Normal-middle: $93.8 \pm 12.2 \mathrm{mg} / \mathrm{dL}$ ).

\section{Multiple analysis}

Table 2 shows the estimates for the components of metabolic syndrome. " $\beta$ " presents standardized regression coefficient and "S.E" presents standardized error of a correlation coefficient. After controlling for covariates, the results showed a positive association between FT4 and fasting glucose level in patients with high FT4 levels when compared to those with normal-middle FT4 levels ( $\beta=15.992 ; p=<0.0001)$. We also identified a significant negative association between FT4 levels and triglycerides in patients with normal-high $(\beta=-21.145, p=0.0054)$ and those with high FT4 levels $(\beta=-49.713, p=0.0404)$. In addition, a positive association was observed between TPOab and triglycerides levels in patients with high TPOab levels in the presence of low median FT4 levels $(\beta=36.075, p=0.0247)$. Also, positive association was identified between TPOab and triglycerides levels in patients with high TPOab levels in the presence of high median TSH levels ( $\beta=32.181, p=0.0368$ ).

\section{Sensitivity analysis}

Table 3 shows the subgroup analysis of the association between the components of metabolic syndrome and FT4 hormone levels according to age and gender. When stratified by age, a significant negative association between FT4 and triglycerides was observed among patients aged 19-44 years (low: $\beta=124.396, p=0.0165$; normal-high: $\beta=-25.519, p=0.0050)$. This association was stronger in the 19-44-year-old age group than in the other older age groups.

Additional file 1: Table S1 presents the subgroup analysis of the association between the components of metabolic syndrome and TSH levels stratified by age and gender. Additional file 1: Table S2 shows the subgroup analysis of the association between the components of metabolic syndrome and TPOab levels with categorizing FT4 levels stratified by age and gender. In addition, Additional file 1: Table S3 shows the subgroup analysis of the association between the components of metabolic syndrome and TPOab levels with categorizing TSH levels stratified by age and gender. TSH was not significantly associated with other components of metabolic syndrome, except HDL cholesterol. Additional file 1: Table $\mathrm{S} 2$ shows that a positive association between TPOab and triglycerides levels is more frequently observed in male with high TPOab titers but with low FT4 levels $(\beta=136.104, p=0.0062)$. 


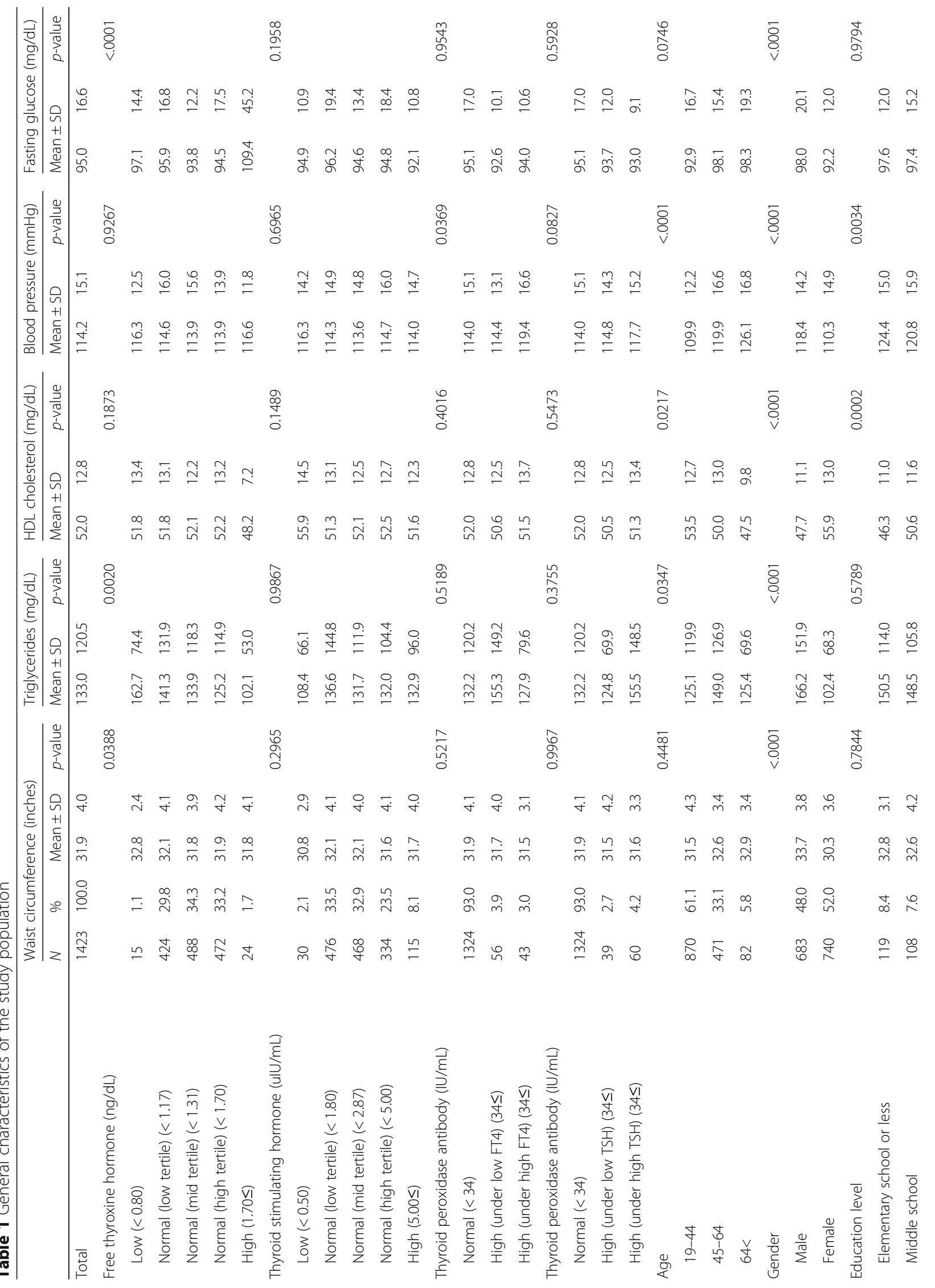




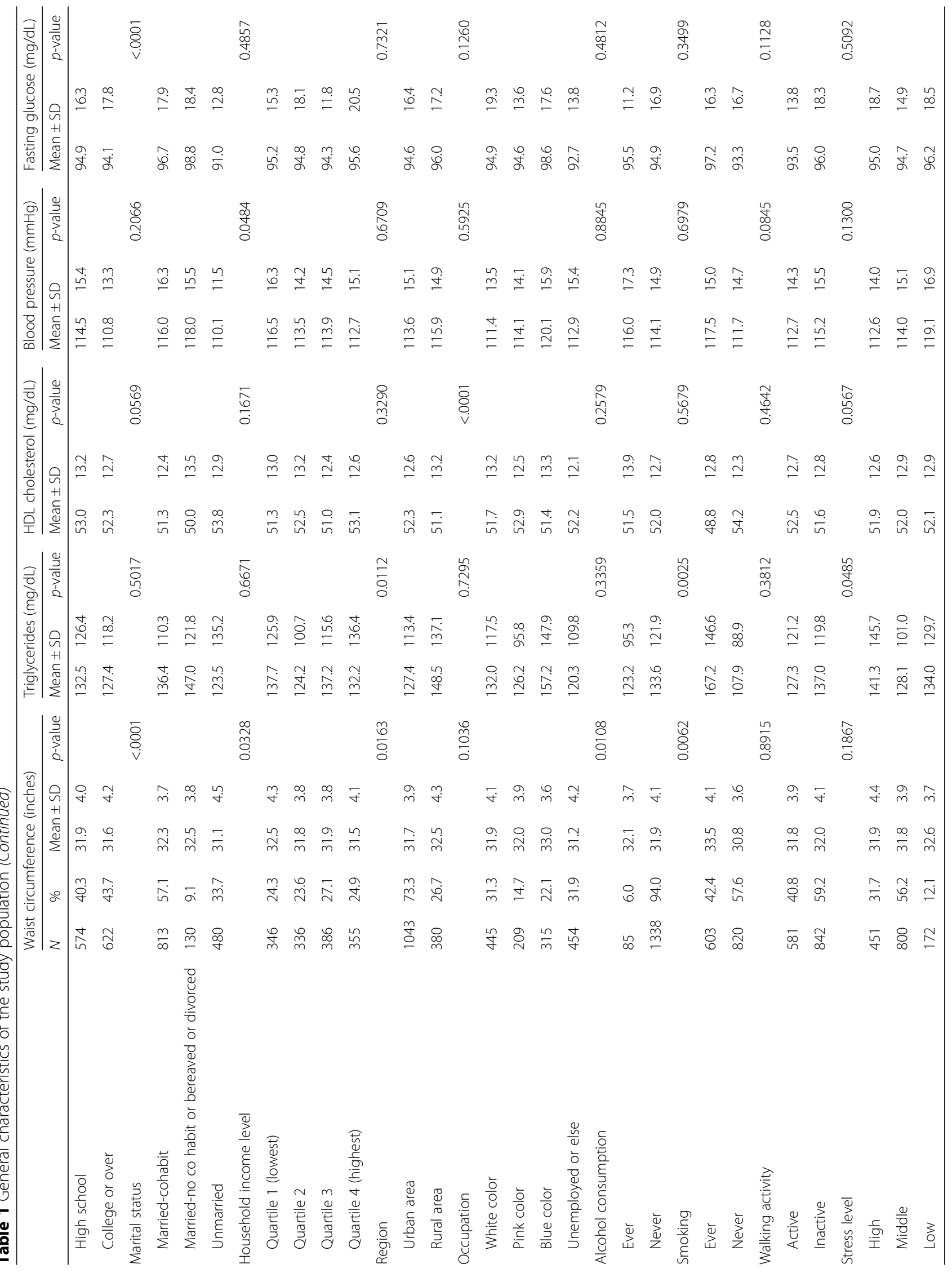




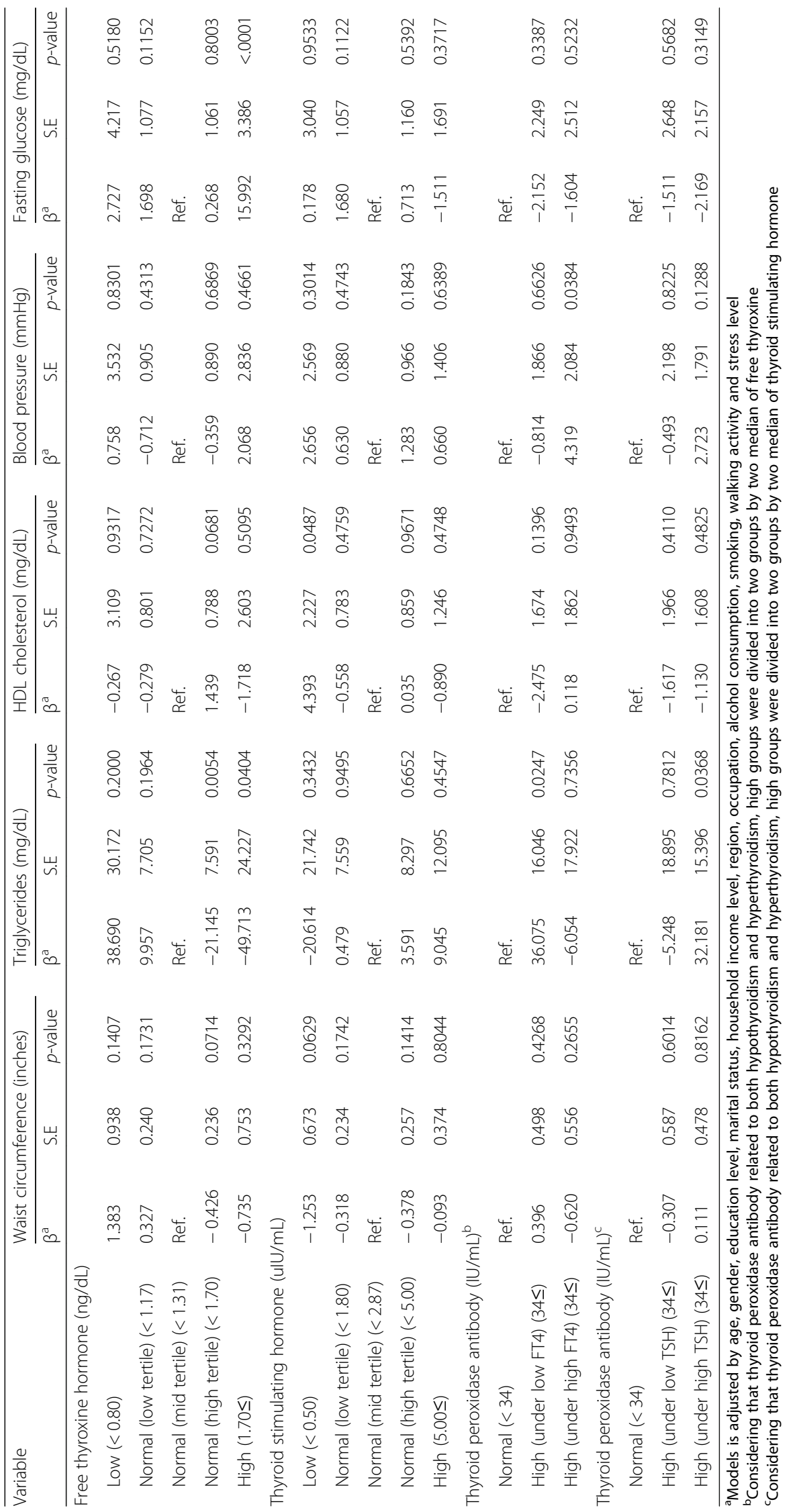


Table 3 Subgroup analysis of components of metabolic syndromes with free thyroxine hormone levels stratified by age and gender

\begin{tabular}{|c|c|c|c|c|c|c|c|c|c|c|c|c|c|}
\hline \multirow[t]{3}{*}{ Variables } & \multicolumn{13}{|c|}{ Free thyroxine hormone levels } \\
\hline & \multicolumn{3}{|l|}{ Low } & \multicolumn{3}{|c|}{ Normal (low tertile) } & \multirow{2}{*}{$\begin{array}{l}\text { Normal } \\
\text { (mid tertile) } \\
\beta^{\text {a }}\end{array}$} & \multicolumn{3}{|c|}{ Normal (high tertile) } & \multicolumn{3}{|l|}{ High } \\
\hline & $\overline{\beta^{a}}$ & S.E & $\overline{p \text {-value }}$ & $\overline{\beta^{a}}$ & S.E & $\overline{p \text {-value }}$ & & $\overline{\beta^{a}}$ & S.E & $\overline{p \text {-value }}$ & $\overline{\beta^{a}}$ & S.E & $\overline{p \text {-value }}$ \\
\hline \multicolumn{14}{|c|}{ Waist circumference } \\
\hline \multicolumn{14}{|l|}{ Age } \\
\hline $19 \sim 44$ & 3.248 & 1.715 & 0.0586 & 0.400 & 0.345 & 0.2471 & Ref. & -0.537 & 0.300 & 0.0738 & -1.062 & 0.892 & 0.2343 \\
\hline $45 \sim 64$ & 0.745 & 1.113 & 0.5037 & 0.470 & 0.352 & 0.1818 & Ref. & 0.078 & 0.410 & 0.8495 & 0.394 & 1.668 & 0.8134 \\
\hline $64<$ & -1.085 & 3.667 & 0.7684 & -0.026 & 0.927 & 0.9777 & Ref. & -1.638 & 1.226 & 0.1868 & -3.990 & 3.705 & 0.2859 \\
\hline \multicolumn{14}{|l|}{ Gender } \\
\hline Male & 1.998 & 2.172 & 0.3580 & 0.804 & 0.405 & 0.0477 & Ref. & -0.270 & 0.336 & 0.4206 & -0.684 & 0.957 & 0.4749 \\
\hline Female & 1.481 & 0.982 & 0.1322 & 0.070 & 0.284 & 0.8061 & Ref. & -0.556 & 0.328 & 0.0909 & -2.002 & 1.277 & 0.1173 \\
\hline \multicolumn{14}{|c|}{ Triglycerides } \\
\hline \multicolumn{14}{|l|}{ Age } \\
\hline $19 \sim 44$ & 124.396 & 51.798 & 0.0165 & 10.341 & 10.430 & 0.3217 & Ref. & -25.519 & 9.066 & 0.0050 & -49.843 & 26.938 & 0.0646 \\
\hline $45 \sim 64$ & 22.880 & 41.955 & 0.5858 & 14.699 & 13.253 & 0.2680 & Ref. & -11.085 & 15.441 & 0.4732 & -78.026 & 62.873 & 0.2153 \\
\hline $64<$ & -29.035 & 77.925 & 0.7108 & -6.737 & 19.693 & 0.7335 & Ref. & 20.424 & 26.056 & 0.4363 & -21.240 & 78.744 & 0.7883 \\
\hline \multicolumn{14}{|l|}{ Gender } \\
\hline Male & -8.759 & 88.150 & 0.9209 & 19.926 & 16.443 & 0.2260 & Ref. & -29.603 & 13.619 & 0.0301 & -56.536 & 38.816 & 0.1457 \\
\hline Female & 59.246 & 19.538 & 0.0025 & 6.175 & 5.645 & 0.2744 & Ref. & -8.730 & 6.527 & 0.1815 & -39.210 & 25.395 & 0.1230 \\
\hline \multicolumn{14}{|c|}{ HDL cholesterol } \\
\hline \multicolumn{14}{|l|}{ Age } \\
\hline $19 \sim 44$ & -3.987 & 5.399 & 0.4605 & 0.135 & 1.096 & 0.9021 & Ref. & 2.554 & 0.952 & 0.0075 & -0.867 & 2.954 & 0.7692 \\
\hline $45 \sim 64$ & 0.799 & 4.276 & 0.8518 & -1.313 & 1.360 & 0.3348 & Ref. & -1.687 & 1.581 & 0.2868 & -5.880 & 6.408 & 0.3594 \\
\hline $64<$ & 6.579 & 10.435 & 0.5309 & -1.360 & 2.688 & 0.6149 & Ref. & 3.663 & 3.507 & 0.3007 & 10.076 & 10.581 & 0.3450 \\
\hline \multicolumn{14}{|l|}{ Gender } \\
\hline Male & -4.968 & 6.373 & 0.4360 & -0.661 & 1.201 & 0.5824 & Ref. & 1.028 & 0.994 & 0.3017 & 0.343 & 2.981 & 0.9085 \\
\hline Female & 1.003 & 3.743 & 0.7888 & 0.050 & 1.089 & 0.9634 & Ref. & 1.908 & 1.256 & 0.1292 & -3.308 & 4.865 & 0.4968 \\
\hline \multicolumn{14}{|c|}{ Blood pressure } \\
\hline \multicolumn{14}{|l|}{ Age } \\
\hline $19 \sim 44$ & 9.081 & 5.089 & 0.0747 & -0.032 & 1.029 & 0.9751 & Ref. & 0.639 & 0.893 & 0.4741 & 1.560 & 2.646 & 0.5556 \\
\hline $45 \sim 64$ & -4.007 & 5.626 & 0.4767 & -2.552 & 1.779 & 0.1522 & Ref. & -3.081 & 2.075 & 0.1382 & -1.728 & 8.439 & 0.8378 \\
\hline $64<$ & -12.387 & 20.182 & 0.5417 & 5.314 & 5.101 & 0.3017 & Ref. & -2.610 & 6.749 & 0.7003 & 22.083 & 20.395 & 0.2833 \\
\hline \multicolumn{14}{|l|}{ Gender } \\
\hline Male & -12.655 & 7.995 & 0.1139 & 2.073 & 1.497 & 0.1666 & Ref. & -0.438 & 1.239 & 0.7239 & 2.575 & 3.522 & 0.4649 \\
\hline Female & 4.899 & 3.870 & 0.2060 & -2.082 & 1.122 & 0.0638 & Ref. & -0.001 & 1.294 & 0.9996 & 3.303 & 5.030 & 0.5116 \\
\hline \multicolumn{14}{|c|}{ Fasting glucose } \\
\hline \multicolumn{14}{|l|}{ Age } \\
\hline $19 \sim 44$ & -3.880 & 7.421 & 0.6012 & 1.190 & 1.494 & 0.4261 & Ref. & 1.333 & 1.299 & 0.3050 & 17.139 & 3.859 & $<.0001$ \\
\hline $45 \sim 64$ & 5.895 & 5.216 & 0.2591 & 1.833 & 1.648 & 0.2665 & Ref. & -2.049 & 1.920 & 0.2865 & 14.249 & 7.817 & 0.0690 \\
\hline $64<$ & 13.834 & 18.440 & 0.4561 & -0.592 & 4.660 & 0.8994 & Ref. & 1.248 & 6.166 & 0.8403 & 3.026 & 18.633 & 0.8715 \\
\hline Gender & & & & & & & & & & & & & \\
\hline Male & 0.545 & 11.505 & 0.9623 & 3.968 & 2.146 & 0.0649 & Ref. & -0.335 & 1.778 & 0.8506 & 21.236 & 5.066 & $<.0001$ \\
\hline Female & 3.278 & 3.480 & 0.3466 & 0.346 & 1.006 & 0.7307 & Ref. & 1.431 & 1.163 & 0.2187 & 5.173 & 4.524 & 0.2532 \\
\hline
\end{tabular}




\section{Discussion}

The purpose of this study was to identify whether any associations exist between thyroid hormone levels and metabolic syndrome components. Thyroid dysfunction is well known to affect glucose and lipid metabolism; abnormal glucose level and abnormal lipid profile are important factors of metabolic syndrome [17]. In our study, we found that glucose and lipid metabolism were associated with thyroid dysfunction. A significant positive association was observed between fasting glucose and FT4 in patients with high FT4 levels. In terms of lipid metabolism, a negative association between triglycerides and FT4 was observed among people with normal-high or high FT4 levels. In addition, there was a significant positive association between TPOab and triglycerides levels in patients with high thyroid peroxidase levels and with low median FT4 levels.

Thyroid hormones regulate carbohydrate metabolism [18]. They influence the mRNA and protein expression of the glucose transporter 4, AMP-activated protein kinase, and acetyl CoA carboxylase in skeletal muscle [19]. Hyperthyroidism usually occurs when high FT4 levels leads to its increased production and absorption from glycogen, lactic acid, glycerol, and amino acids [20]. Hyperthyroidism can also increase insulin degradation. Which deteriorates blood glucose control $[19,20]$. Based on these mechanisms, other studies also showed that glucose levels are high in patients with hyperthyroidism [21]. Foss et al. demonstrated that patients with hyperthyroidism had higher blood glucose levels than the euthyroid participants, indicating increased endogenous glucose production [22]. Moreover, Roubsanthisuk et al. reported that the higher glucose intolerance showed common in hyperthyroidism compare to the normal participants [23]. This could be a major cause of the increased risk for diabetes [23]. Health professionals should conduct follow-up tests of the glucose level of patients with high thyroid hormone levels. Additionally, thyroid hormone levels should be checked regularly in patients with high glucose levels.

A significant negative association between FT4 levels and triglycerides was observed in patients with high-normal or high FT4 levels. To explain this, we presumed that thyroid hormones play a key role in the regulation of enzyme activity during lipoprotein transport [12]. The influence of thyroid function on lipid metabolism involves a pathophysiological process [24]. The negative association observed between triglycerides and thyroid hormones can be explained by the increased removal rate of triglycerides from plasma due to an increase in the activity of hepatic triglyceride lipase $[25,26]$. The effects of thyroid hormone on lipid metabolism are well known [26]. Because the lipid profile tends to normalize improperly under high thyroid hormone levels, the lipid profile needs to be followed up after adjusting for thyroid hormone levels [25].
There was a significant positive association between TPOab and triglycerides levels in patients with high thyroid peroxidase levels but with low median FT4 levels. In addition, a positive association was identified between TPOab and triglycerides levels in patients with high thyroid peroxidase levels in the presence of high median TSH levels. The presence of TPOab in the blood indicates a high risk for thyroid disease due to autoimmune disorders [27, 28]. In fact, abnormal TPOab levels were observed in $90 \%$ of patients with hypothyroidism and Graves' disease [27, 28]. Therefore, the positive association between TPOab and triglycerides levels in patients with high thyroid peroxidase levels but low median FT4 levels may be related to hypothyroidism.

Based on the results of the subgroup analysis, a significant negative association was observed between FT4 and triglyceride levels in patients aged 19-44 years; this association was stronger in the 19-44-year-old age group than in the other older age groups. This finding emphasizes the possibility of accumulation of risk through one's life course. Accumulation of various risks increases owing to illness, health-damaging behaviors, and adverse environmental conditions [29]. Older people may have more risk factors owing to their risk accumulation, and are more likely to have unmeasured risk factors that we could not adjust for [30]. To address this issue, future studies developed to understand the association between thyroid hormones and metabolic syndrome should incorporate a panel design.

This study had some limitations. First, the study was based on a cross-sectional survey. Causality could not be confirmed clearly and only the association could be confirmed. Second, one of the major thyroid hormones, triiodothyronine, was not used in the thyroid function tests. Despite the above limitations, this study also has a few strengths. First, consistent blood tests showed accurate blood TSH levels. Second, most of the previous studies were conducted in Caucasians; therefore, this issue is worth pursuing among Koreans. Finally, this study used the most recent (KNHANES 2013-2015) nationally, multistage, stratified collected data and could therefore be considered to be representative of the Korean population.

\section{Conclusion}

FT4 showed a partially positive association with fasting glucose, and a partially negative association with triglycerides in the general Korean population. Glucose level and lipid profile are metabolic syndrome components; metabolic syndrome is strongly correlated with diseases such as diabetes and cardiovascular disease that are associated with high mortality and morbidity rates. In patients with abnormal thyroid function, follow up tests for metabolic syndrome components during thyroid dysfunction could prevent overlooking metabolic syndrome and prevent related diseases. 


\section{Additional file}

Additional file 1: Table S1. Subgroup analysis of components of metabolic syndromes with thyroid stimulating hormone levels stratified by age and gender. Table S2. Subgroup analysis of components of metabolic syndromes with thyroid peroxidase antibody levels stratified by age and gender. Table S3. Subgroup analysis of components of metabolic syndromes with thyroid peroxidase antibody levels stratified by age and gender. (DOCX $88 \mathrm{~kb}$ )

\section{Abbreviations}

Cl: Confidence interval; FT4: Free thyroxine; KNHANES: Korea National Health and Nutrition Examination Surveys; OR: Odds ratio; TPOab: Thyroid peroxidase antibody; TSH: Thyroid-stimulating hormone

\section{Acknowledgements}

We appreciate Department of Health and Human Services Centers for Disease Control and Prevention that provided meaningful data.

\section{Availability of data and materials}

The datasets generated and/or analysed during the current study are available in the KNHNES website (http://www.cdc.go.kr/CDC/contents/CdcKrContent View.jsp?cid=60940\&menulds=HOME001-MNU1130-MNU1639-MNU1748MNU1752). The KNHANES was opened in https://knhanes.cdc.go.kr/knhanes/ index.do after submitting written oath and data utilization plan.

\section{Authors' contributions}

E.-C.P. (corresponding author) reviewed the manuscript. J.J. (cofirst author) and Y. K. (cofirst author) wrote the draft of the manuscript and analyzed the data. J. S., S. A. L. and Y. C. provided assistance for the planning, execution, execution, and analysis of the study. All authors read and approved final manuscript. The authors appreciate the administrative support provided by the Yonsei University Institute of Health Services Research. This research study was not funded by any foundation.

\section{Ethics approval and consent to participate}

The Institutional Review Board (IRB) of the Korea Centers for Disease Control and Prevention (KCDC) provided formal ethics approval for the KNHANES data sets (IRB approval number 2013-07CON-03-4C for 2013, 2013-12EXP-035C for 2014, 2015-01-02-6C for 2015).

\section{Competing interests}

The authors declare that they have no competing interests.

\section{Publisher's Note}

Springer Nature remains neutral with regard to jurisdictional claims in published maps and institutional affiliations.

\section{Author details}

${ }^{1}$ Department of Public Health, Graduate School, Yonsei University, Seoul, Republic of Korea. ${ }^{2}$ Institute of Health Services Research, Yonsei University, Seoul, Republic of Korea. ${ }^{3}$ Department of Anesthesia, Indiana University School of Medicine, Indianapolis 46202, USA. ${ }^{4}$ Department of Preventive Medicine \& Institute of Health Services Research, Yonsei University College of Medicine, 50 Yonsei-ro, Seodaemun-gu, Seoul 120-752, Republic of Korea.

Received: 11 September 2017 Accepted: 3 May 2018

Published online: 21 May 2018

\section{References}

1. Grundy SM. Obesity, metabolic syndrome, and cardiovascular disease. J. Clin. Endocrinol. Metab. 2004;89(6):2595-600.

2. Roos A, Bakker SJ, Links TP, Gans RO, Wolffenbuttel BH. Thyroid function is associated with components of the metabolic syndrome in euthyroid subjects. J. Clin. Endocrinol. Metab. 2007:92(2):491-6.

3. Ramachandran A, Snehalatha C, Satyavani K, Sivasankari S, Vijay V. Metabolic syndrome in urban Asian Indian adults - a population study using modified ATP III criteria. Diabetes Res Clin Pract. 2003;60(3):199-204.
4. Aguilar M, Bhuket T, Torres S, Liu B, Wong RJ. Prevalence of the metabolic syndrome in the United States, 2003-2012. JAMA. 2015;313(19):1973-4.

5. Vishram JK, Borglykke A, Andreasen AH, Jeppesen J, Ibsen H, Jørgensen T, Palmieri L, Giampaoli S, Donfrancesco C, Kee F. Impact of age and gender on the prevalence and prognostic importance of the metabolic syndrome and its components in Europeans. The MORGAM prospective cohort project. PLoS One. 2014;9(9):e107294.

6. Park S, Kim S-J, Lee M, Kang K-A, Hendrix E. Prevalence and associated factors of metabolic syndrome among south Korean adults. J Community Health Nurs. 2015;32(1):24-38.

7. Korea S: Cause of death statistics. Statistics Korea 2016

8. Mullur R, Liu Y-Y, Brent GA. Thyroid hormone regulation of metabolism. Physiol Rev. 2014;94(2):355-82.

9. Lambadiari V, Mitrou P, Maratou E, Raptis AE, Tountas N, Raptis SA, Dimitriadis G. Thyroid hormones are positively associated with insulin resistance early in the development of type 2 diabetes. Endocrine. 2011;39(1):28-32.

10. Dimitriadis $G$, Raptis $S$. Thyroid hormone excess and glucose intolerance. Exp Clin Endocrinol Diabetes. 2001;109(Suppl 2):S225-39.

11. Klein I, Ojamaa K. Thyroid hormone and the cardiovascular system. N Engl J Med. 2001;344(7):501-9.

12. Duntas LH. Thyroid disease and lipids. Thyroid. 2002;12(4):287-93.

13. Ruhla S, Weickert MO, Arafat AM, Osterhoff M, Isken F, Spranger J, Schöfl C, Pfeiffer AF, Möhlig M. A high normal TSH is associated with the metabolic syndrome. Clin Endocrinol. 2010;72(5):696-701.

14. Yang $\mathrm{M}, \mathrm{Qu} \mathrm{H}$, Deng $\mathrm{H}-\mathrm{C}$. Acute pancreatitis induced by methimazole in a patient with Graves' disease. Thyroid. 2012:22(1):94-6.

15. Davies PH, Franklyn JA, Daykin J, Sheppard MC. The significance of TSH values measured in a sensitive assay in the follow-up of hyperthyroid patients treated with radioiodine. J. Clin. Endocrinol. Metab. 1992;74(5): 1189-94.

16. Goswami R, Goel S, Tomar N, Gupta N, Lumb V, Sharma YD. Prevalence of clinical remission in patients with sporadic idiopathic hypoparathyroidism. Clin Endocrinol. 2010;72(3):328-33.

17. Rizos C, Elisaf M, Liberopoulos E. Effects of thyroid dysfunction on lipid profile. Open Cardiovasc. Med. J. 2011;5:76.

18. de Jesus G-GJ, Alvirde-Garcia U, Lopez-Carrasco G, Mendoza MEP, Mehta R, Arellano-Campos O, Choza R, Sauque L, Garay-Sevilla ME, Malacara JM. TSH and free thyroxine concentrations are associated with differing metabolic markers in euthyroid subjects. Eur J Endocrinol. 2010;163(2):273-8.

19. Crunkhorn S, Patti M-E. Links between thyroid hormone action, oxidative metabolism, and diabetes risk? Thyroid. 2008;18(2):227-37.

20. Bhattacharyya A, Wiles P. Diabetic ketoacidosis precipitated by thyrotoxicosis. Postgrad Med J. 1999;75(883):291-3.

21. Gu Y, Li H, Bao X, Zhang Q, Liu L, Meng G, Wu H, Du H, Shi H, Xia Y. The relationship between thyroid function and the prevalence of type 2 diabetes mellitus in euthyroid subjects. J. Clin. Endocrinol. Metab. 2016;jc:2016-965.

22. Foss MC, Paccola GM, Saad MJ, Pimenta WP, Piccinato CE, lazigi N. Peripheral glucose metabolism in human hyperthyroidism. J. Clin. Endocrinol. Metab. 1990;70(4):1167-72.

23. Roubsanthisuk W, Watanakejorn P, Tunlakit M, Sriussadaporn S. Hyperthyroidism induces glucose intolerance by lowering both insulin secretion and peripheral insulin sensitivity. J. Med. Assoc. Thai. = Chotmaihet thangphaet. 2006;89(Suppl 5):S133-40.

24. Park SB, Choi HC, Joo NS. The relation of thyroid function to components of the metabolic syndrome in Korean men and women. J Korean Med Sci. 2011;26(4):540-5.

25. Pucci E, Chiovato L, Pinchera A. Thyroid and lipid metabolism. Int J Obes. 2000;24(S2):S109.

26. Tulloch B, Lewis B, Fraser TR. Triglyceride metabolism in thyroid disease. Lancet. 1973;301(7800):391-4.

27. Sultana Q, Anjum A, Fathima N, Siraj M, Ishaq M. Seropositivity to antithyroid peroxidase and anti-thyroglobulin autoantibodies in hypo and hyper-thyroidism: Diagnostic and epidemiological significance. IJMR. 2016; 3(4):368-72.

28. Swain M, Swain T, Mohanty BK. Autoimmune thyroid disorders-an update. Indian J Clin Biochem. 2005;20(1):9-17.

29. Kuh D, Ben-Shlomo Y, Lynch J, Hallqvist J, Power C. Life course epidemiology. J Epidemiol Community Health. 2003;57(10):778.

30. Organization WH: Life course perspectives on coronary heart disease, stroke and diabetes: key issues and implications for policy and research: summary report of a meeting of experts, 2-4 may 2001. 2001. 